\title{
On the Theory of Dendritic Growth: Soret and Temperature-Dependent Diffusion Effects
}

\author{
D. V. Alexandrov* and D. A. Pinigin \\ Ural Federal University, ul. Mira 19, Yekaterinburg, 620002 Russia \\ *e-mail: Dmitri.Alexandrov@usu.ru \\ Received April 27, 2012
}

\begin{abstract}
An analytical solution is found for the problem of the growth of an isolated dendrite in a convective binary melt with allowance for the Soret and temperature-dependent diffusion effects. Nonlinear impurity transport is shown to radically change the impurity concentration in front of the growing crystal and, correspondingly, the concentration supercooling, which is responsible for the condition of choosing the dendrite tip growth rate.
\end{abstract}

DOI: $10.1134 / \mathrm{S} 0036029513020031$

\section{INTRODUCTION}

The evolution of dendrites is known to control the structure formation in the materials formed during the solidification of melts [1, 2]. Apart from experimental observation of the dendritic growth dynamics, qualitatively new materials have recently been obtained by mathematical simulation to test the basic concepts of crystal morphology formation (see review [3] and Refs. therein). Among the important problems, we distinguish the problem of stable growth of the tip of a freely growing dendrite crystal and the problem of the effect of a convective flow on the mechanism of choosing its growth mode. These problems have theoretical and practical importance $[4,5]$.

The problem of choosing the stable growth mode of an isolated crystal appeared from an analysis of the Ivantsov solutions $[6,7]$ and the experimental data on the growth of a needlelike crystal of a parabolic shape [8-14]. These comparison and tests led to the conclusion that the continuous family of isotropic Ivantsov solutions is unstable: a needlelike crystal loses its initial parabolic shape in a steady-state growth mode [15]. Therefore, the Ivantsov solution is only used as a zeroth approximation to solve the problem of stable growth, where the role of a small parameter is played by surface tension anisotropy or growth kinetics anisotropy [16]. After finding the criterion of stable dendrite tip motion in a single-component unmovable medium $[15,16]$, the problem was extended to the cases of a convective medium flow [17-19], crystal growth in a binary (chemically two-component) convective-free system [20], and dendritic growth in a binary liquid with convection [21, 22].

Directional solidification is known to be controlled by changing the temperature gradients or temperatures in solid and liquid phases. The temperature gradients and temperatures set far from the phase-transi- tion temperature can be rather high. This circumstance can result in the thermodiffusion effect (Soret effect), when the difference in the temperatures in various melt regions leads to substance transport into them; that is, a temperature gradient changes the concentration (see, e.g., [23]). As a rule, a thermodiffusion flow depends on the substance concentration. For example, it is proportional to the impurity concentration in dilute solutions and melts [23-25]. Therefore, we can write the substance flow in the form

$$
\vec{J}=-D \nabla C-D_{T}\left(\beta_{0}+\beta_{1} C\right) \nabla T,
$$

where $C$ and $T$ are the concentration and temperature fields, respectively; $D$ and $D_{T}$ are the diffusion and thermodiffusion coefficients, respectively; and $\beta_{0}$ and $\beta_{1}$ are constants. The first term in Eq. (1) is responsible for conventional substance diffusion, and the second term, for thermodiffusion. We will consider the following cases: $\beta_{0}=C_{\infty}, \beta_{1}=0$ ( $C_{\infty}$ is the initial concentration, or the concentration at infinity) and $\beta_{0}=0$, $\beta_{1}=1$. The first case was discussed in [26]: it corresponds to small concentration changes as a function of the spatial coordinate in a melt. If the change in the concentration cannot be neglected, the second case is better to describe the process [27].

The inverse Soret effect (so-called Dufour effect), where a concentration gradient causes temperature changes, is also known. However, when solutions and melts are studied, this effect may be neglected (as a rule, it is important in gas mixtures (see, e.g., [28])).

To facilitate calculations, the diffusion coefficient is usually taken to be constant; however, generically speaking, this is not the case, since the diffusion coefficient depends on temperature. By analogy with 


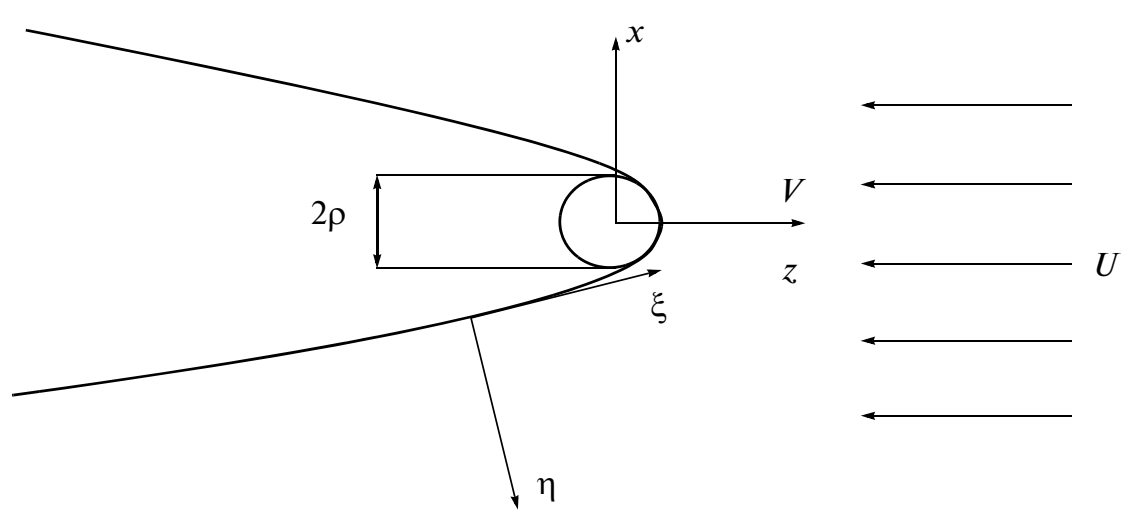

Fig. 1. Schematic diagram of dendrite growth in an incident liquid flow.

$[27,29]$, we consider this temperature dependence in the linear form

$$
D(T)=D_{0}+\frac{\partial D}{\partial T}\left(T-T_{0}\right),
$$

where $D_{0}$ is the diffusion coefficient at melting temperature $T_{0}$ and $\partial D / \partial T=D^{\prime}$ is the temperature coefficient (see, e.g., [29]).

It is known from experiments that the growth of dendritic structures completely characterizes the properties of solids. Solidification usually proceeds in the mode of "dendritic forest" formation in the supercooled region in a melt, which is often called a mushy zone $[30,31]$. Therefore, to study the specific features of dendritic growth is important from the standpoint of structure-phase transitions in the mushy zone. The rejection of impurities by a growing crystal (dendrites) is known to result in a concentration supercooling [32], which favors the growth of a solid phase deep into the melt. The supercooling that appears during the solidification of metallic alloys is often compensated by intense formation of latent heat of solidification. In this case, the mushy zone having appeared during solidification is quasi-equilibrium [33-35].

The authors of [36-43] developed methods for solving nonlinear heat- and mass-transfer equations in a quasi-equilibrium mushy zone solidifying at a constant rate. This process takes place in many experiments and corresponds to the pulling of a crystal from a melt. Solidification often occurs from a cooling boundary, the temperature of which is maintained at a constant level or is a function of time. In the former case, solidification corresponds to a self-similar mode. The theory of mushy zone solidification for this situation was developed in [44-52]. In the latter case, the system dynamics depends substantially on the time variation of the cooling boundary temperature. To describe the processes that take place under these conditions, the authors of [53-65] developed methods for analytical solution of quasi-equilibrium mushy zone equations.
Thermodiffusion and the temperature dependence of a diffusion coefficient during the heat and mass transfer in the mushy zone were taken into account in $[66,67]$. In those works, however, the mushy zone was considered without taking into account the specific features of the growth of individual dendritic structures; therefore, it is important to perform such an analysis. Although dendritic growth was studied in many works, the temperature dependence of a diffusion coefficient and the possibility of thermodiffusion substance transport have not yet been considered. The purpose of this work is study the effect of Eqs. (1) and (2) on the impurity distribution created by the growth of an isolated dendrite in an incident melt flow.

The problem is formulated for an extended Stefan model with a frontal interfacial dendrite surface (parabolic crystal-liquid interface) when thermodiffusion and the temperature dependence of a diffusion coefficient are taken into account. The problem for a forced flow is solved in the Oseen approximation.

\section{MODEL FOR DENDRITIC GROWTH}

Figure 1 shows the dendritic growth in an incident viscous melt flow. The temperature field in the liquid phase is described by the convective heat conduction equation

$$
\frac{\partial T}{\partial t}+(\vec{w}, \nabla) T=a \Delta T
$$

where $a$ is the thermal diffusivity, $\vec{w}$ is the liquid flow velocity, $t$ is the time, and $\Delta$ is the Laplace operator.

The impurity distribution in the liquid part of the system obeys the convective diffusion equation

$$
\frac{\partial C}{\partial t}+(\vec{w}, \nabla) C=-\nabla \vec{J}
$$

and flow $\vec{J}$ is determined by Eq. (1).

The heat and mass balance conditions

$$
\begin{gathered}
Q \overrightarrow{\mathrm{v}} \cdot \vec{n}=a c_{\mathrm{p}}\left(\nabla T_{\mathrm{s}}-\nabla T\right) \vec{n}, \\
\left(1-k_{0}\right) C \overrightarrow{\mathrm{v}} \cdot \vec{n}-\vec{J} \vec{n}=0 .
\end{gathered}
$$


hold true at the mobile interface. Here, $Q$ is the latent heat of solidification, $T_{\mathrm{s}}$ is the (isothermal) dendrite temperature, $\vec{v} \vec{n}$ is the normal velocity of the dendrite surface, $c_{\mathrm{p}}$ is the heat capacity of the melt, and $k_{0}$ is the equilibrium impurity distribution coefficient. Following [19], we consider the liquid flow at small Reynolds numbers. In this case, the velocity distribution in the melt obeys the Oseen equation and the continuity equation [68]

$$
U \frac{\partial \vec{w}}{\partial z}=-\frac{1}{\rho_{1}} \nabla p+v \Delta \vec{w}, \quad \nabla \vec{w}=0,
$$

where $U$ is the incident liquid flow velocity far from the growing crystals and $\rho_{1}$ and $v$ are the liquid density and viscosity, respectively. Note that the Oseen approximation used in motion equation (7) can only take into account the most important inertial terms and yields rather accurate calculation results (see, e.g., the classical problem of the motion of a sphere in a viscous liquid $[69,70])$.

\section{ANALYTICAL SOLUTION}

Let a two-dimensional parabolic dendrite grow at a constant rate $V$ along spatial axis $z$ (Fig. 1). The liquid flow far from the crystal is parallel to axis $z$ and is opposite to the dendrite growth direction (so-called incident flow). We introduce parabolic coordinates $\xi$ and $\eta$ connected to Cartesian coordinates $x$ and $z$ by the relations

$$
x=\rho \sqrt{\xi \eta}, \quad z=\frac{\rho}{2}(\eta-\xi),
$$

where $\rho$ is the dendrite tip radius and the interface is located at the level $\eta=1$.

With Eq. (7), we can determine liquid velocity components $u_{\eta}$ and $u_{\xi}$ in parabolic coordinates (8). Following [19], we write the result in the form

$$
u_{\eta}=-\frac{f(\eta)}{2 \sqrt{\xi+\eta}}, \quad u_{\xi}=\frac{\sqrt{\xi \eta}}{\sqrt{\xi+\eta}} \frac{d f}{d \eta}
$$

where we introduced the defining functions

$$
\begin{gathered}
f(\eta)=2(U+V) \sqrt{\eta}-2 U g(\eta), \\
g(\eta)=\sqrt{\eta} \frac{\operatorname{erfc} \sqrt{\eta \operatorname{Re} / 2}}{\operatorname{erfc} \sqrt{\operatorname{Re} / 2}}+\frac{\sqrt{2 /(\pi \operatorname{Re})}}{\operatorname{erfc} \sqrt{\operatorname{Re} / 2}} \\
\times[\exp (-\operatorname{Re} / 2)-\exp (-\eta \operatorname{Re} / 2)],
\end{gathered}
$$

which take into account the flow intensity using Reynolds number $\operatorname{Re}=\rho_{1} U / v$.

Equation (3) can easily be integrated in parabolic coordinates (8). Having found its solution (which depends only on variable $\eta$ ), we rewrite the formulation of the problem in the form (see [18])

$$
\begin{gathered}
u_{\eta} \frac{d T}{d \eta}=\frac{2 a}{\rho \sqrt{\xi+\eta}}\left(\sqrt{\eta} \frac{d^{2} T}{d \eta^{2}}+\frac{1}{2 \sqrt{\eta}} \frac{d T}{d \eta}\right), \\
\left(\frac{d T}{d \eta}\right)_{\eta=1}=-\frac{Q}{c_{\mathrm{p}}} \frac{\rho V}{2 a},
\end{gathered}
$$

where the temperature in the melt at infinity is considered to be specified; that is, $T \rightarrow T_{\infty}$ at $\eta \rightarrow \infty$.

The integration of Eq. (10) gives the temperature distribution in the liquid phase of the system,

$$
T(\eta)=T_{i}+\left(T_{\infty}-T_{i}\right) \frac{I(\eta)}{I(\infty)} .
$$

Here, we introduced the following designations:

$$
\begin{gathered}
I(\eta)=\int_{1}^{\eta} \exp \left[P_{f} \int_{1}^{\eta^{\prime}} \frac{g\left(\eta^{\prime \prime}\right)}{\sqrt{\eta^{\prime \prime}}} d \eta^{\prime \prime}-\left(P_{f}+P_{g}\right) \eta^{\prime}\right] \frac{d \eta^{\prime}}{\sqrt{\eta^{\prime}}}, \\
T_{i}=T_{\infty}+\frac{Q}{c_{\mathrm{p}}} P_{g} \exp \left(P_{f}+P_{g}\right) I(\infty), P_{g}=\frac{\rho V}{2 a}, P_{f}=\frac{\rho U}{2 a},
\end{gathered}
$$

where $P_{g}$ and $P_{f}$ are the growth and flow Peclet numbers, respectively, which are defined in terms of dendrite growth rate $V$ and liquid flow velocity $U$.

Equation (4) depends only on coordinate $\eta$ and is written as

$$
\begin{gathered}
-\frac{f(\eta)}{2} \frac{d C}{d \eta}=\frac{2}{\rho} \frac{d}{d \eta} \\
\times\left[\sqrt{\eta}\left(D(T) \frac{d C}{d \eta}+D_{T}\left(\beta_{0}+\beta_{1} C\right) \frac{d T}{d \eta}\right)\right] .
\end{gathered}
$$

We introduce designations $y(\eta)=\beta_{0}+\beta_{1} C(\eta)$ and $d y / d \eta=y(\eta) / q(\eta)$ and reduce the order of Eq. (12),

$$
\frac{d q}{d \eta} \cong F(q, \eta)
$$

where

$$
\begin{aligned}
& F(q, \eta)=-\frac{D_{T} \beta_{1}}{D(T)}\left[\frac{1}{2 \eta} \frac{d T}{d \eta}+q \frac{d T}{d \eta}+\frac{d^{2} T}{d^{2} \eta}\right]-q^{2} \\
& -\frac{D_{T}}{D_{0}} \frac{P_{f}+P_{g}-P_{f} g(\eta) / \sqrt{\eta}}{1+\left(T(\eta)-T_{0}\right) D^{\prime} / D_{0}} q-\frac{q D^{\prime}}{D(T)} \frac{d T}{d \eta}-\frac{q}{2 \eta} .
\end{aligned}
$$

Here, the temperature and its derivative are known and determined with Eq. (11), and the ratios of coefficients $D_{T} / D(T)$ and $D^{\prime} / D(T)$ are

$$
\begin{aligned}
& \frac{D_{T}}{D(T)}=\frac{D_{T} / D_{0}}{1+\left(T-T_{0}\right) D^{\prime} / D_{0}}, \\
& \frac{D^{\prime}}{D(T)}=\frac{D^{\prime} / D_{0}}{1+\left(T-T_{0}\right) D^{\prime} / D_{0}} .
\end{aligned}
$$




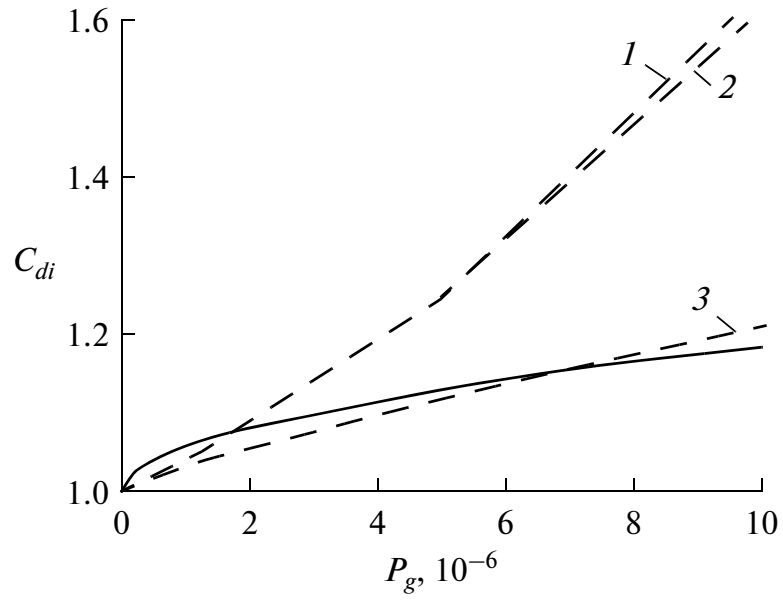

Fig. 2. Impurity concentration on the dendrite surface $C_{d i}$ (at $\eta=1$ ) vs. Peclet number $P_{g}$. (solid line) Solution to the problem in the absence of the Soret and temperaturedependent diffusion effects $\left(D_{T}=D^{\prime}=0, D=D_{0}=\right.$ const $)$. (dashed lines) Calculation at (1) $D_{T} / D_{0}=-10^{-2} \mathrm{~K}^{-1}$, $D^{\prime} / D_{0}=10^{-1} \mathrm{~K}^{-1}$; (2) $D_{T} / D_{0}=10^{-1} \mathrm{~K}^{-1}, D^{\prime} / D_{0}=10^{-1} \mathrm{~K}^{-1}$; and (3) $D_{T} / D_{0}=-10^{-2} \mathrm{~K}^{-1}, D^{\prime} / D_{0}=1 \mathrm{~K}^{-1}$.

Boundary condition (6) on the dendrite surface $\eta=1$ is now written in the situation where $\beta_{0}=0$ and $\beta_{1}=1[27]$,

$$
q=-\frac{D_{T} / D_{0}}{1+\left(T-T_{0}\right) D^{\prime} / D_{0}} \frac{d T}{d \eta}-\frac{\left(1-k_{0}\right) P_{g} D_{T} / D_{0}}{1+\left(T-T_{0}\right) D^{\prime} / D_{0}} .
$$

Equations (13) and (14) represent the Cauchy problem the solution to which determines function $q(\eta)$. Using the found dependence $q(\eta)$, we can determine the impurity concentration distribution in the melt,

$$
C(\eta)=\frac{1}{\beta_{1}}\left[\left(\beta_{0}+\beta_{1} C_{\infty}\right) \frac{\exp \left(\int_{1}^{\eta} q(\lambda) d \lambda\right)}{\exp \left(\int_{1}^{\infty} q(\lambda) d \lambda\right)}-\beta_{0}\right],
$$

where $C_{\infty}$ is the impurity concentration in the melt far from the dendrite. Thus, Eqs. (11) and (13)-(15) represent the solution to the heat-and-mass transfer problem of dendritic growth when the thermodiffusion and temperature-dependent diffusion effects are taken into account.

\section{CONCLUSIONS}

Figure 2 shows the impurity concentration on the dendrite surface versus the growth Peclet number. The calculation parameters of the system were borrowed from [21, 22, 66, 67]: $a / D_{0}=5 \times 10^{3}, k_{0}=0.5, C_{\infty}=1$, $Q / c_{\mathrm{p}}=300, a / v=10, P_{f}=10^{-6}, T_{\infty}=1000^{\circ} \mathrm{C}, \beta_{0}=0$, and $\beta_{1}=1$. An analysis of the calculated curves demonstrates that the presence of nonlinear impurity transport effects in the melt radically changes the impurity distribution. In this case, the role of coefficient $D^{\prime}$ is more substantial than the role of coefficient $D_{T}$ in the system parameter ranges under study.

The change in the surface impurity concentration (and the concentration profile in the melt) results in a change in the concentration supercooling in the space in front of the dendrite tip. The calculations show that the impurity concentration near the dendrite tip can both increase and decrease. As a result, the concentration supercooling increases or decreases, respectively, and the dendrite tip growth rate changes respectively. To study this problem in detail, it is necessary to analyze the stability of dendritic growth with allowance for the Soret and temperature-dependent diffusion effects in terms of works [18-22].

In conclusion, note that the effects of nonlinear impurity transport considered in this work will change the solutions to the equations of a weakly nonequilibrium mushy zone [71-73] and will affect the interface stability [74-84].

\section{ACKNOWLEDGMENTS}

This work was supported by the Russian Foundation for Basic Research (project no. 11-01-00137) and the Federal Target Program "Scientific and AcademicTeaching Staff of Innovative Russia" in 2009-2013.

\section{REFERENCES}

1. W. Kurz and D. J. Fisher, Fundamentals of Solidification (Trans. Tech., Aedermannsdorf, 1992).

2. D. M. Herlach, P. Galenko, and D. Holland-Moritz, Metastable Solids from Undercooled Melts (Elsevier, Amsterdam, 2007).

3. M. Asta, C. Beckermann, A. Karma, W. Kurz, R. Napolitano, M. Plapp, G. Purdy, M. Rappaz, and R. Trivedi, "Solidification microstructures and solidstate parallels: recent developments, future directions," Acta Mater. 57, 941-971 (2009).

4. P. K. Galenko, O. Funke, J. Wang, and D. M. Herlach, "Kinetics of dendritic growth under the influence of convective flow in solidification of undercooled droplets," Mater. Sci. Eng. A 488, 375-377 (2004).

5. O. Funke, G. Phanikumar, P. K. Galenko, L. Chernova, S. Reutzel, M. Kolbe, amnd D. M. Herlach, "Dendrite growth velocity in levitated undercooled nickel melts,” J. Cryst. Growth 297, 211-222 (2006).

6. G. P. Ivantsov, "Temperature field around a spherical, cylindrical, or needlelike dendrite growing in a supercooled melt," Dokl. Akad. Nauk SSSR 58, 567-570 (1947).

7. G. P. Ivantsov, "On the growth of a spherical or a needlelike crystal of a binary alloy," Dokl. Akad. Nauk SSSR 83, 573-576 (1952). 
8. D. E. Temkin, "On the growth of a crystalline needle in a supercooled melt," Dokl. Akad. Nauk SSSR 132, 1307-1310 (1960).

9. D. E. Temkin, "Growth kinetics of a crystalline needle in a supercooled binary melt," Kristallografiya 7, 446450 (1962).

10. G. Horvay and J. W. Cahn, "Dendritic and spheroidal growth,” Acta Metall. 9, 695-705 (1961).

11. W. W. Mullins and R. F. Sekerka, "Stability of a planar interface during solidification of a dilute binary alloy," J. Appl. Phys. 35, 444-451 (1964).

12. G. E. Nash and M. E. Glicksman, "Capillarity-limited steady-state dendritic growth: I. Theoretical development," Acta Metall. 22, 1283-1290 (1974).

13. J. S. Langer and H. Müller-Krunbhaar, "Theory of dendritic growth: I. Elements of a stability analysis," Acta Metall. 26, 1681-1687 (1978).

14. R. Willnecker, D. Herlach, and B. Feuerbacher, "Evidence of nonequilibrium processes in rapid solidification of undercooled metals," Phys. Rev. Lett. 62, 2707-2710 (1989).

15. D. A. Kessler, J. Koplik, and H. Levine, "Pattern selection in fingered growth phenomena," Adv. Phys. 37, 255-339 (1988).

16. E. Brener and V. I. Melnikov, "Pattern selection in twodimensional dendritic growth," Adv. Phys. 40, 53-97 (1991).

17. S. K. Dash and W. N. Gill, "Forced convection heat and momentum transfer to dendritic structures (parabolic cylinders and paraboloids of revolution), " Int. J. Heat Mass Transf. 27, 1345-1356 (1984).

18. M. Ben-Amar, Ph. Bouisou, and P. Pelce, "An exact solution for the shape of a crystal growing in a forced flow," J. Cryst. Growth 92, 97-100 (1988).

19. Ph. Bouissou and P. Pelce, "Effect of a forced flow on dendritic growth,” Phys. Rev. A 40, 6673-6680 (1989).

20. M. Ben Amar and P. Pelce, "Impurity effect on dendritic growth," Phys. Rev. A 39, 4263-4269 (1989).

21. D. V. Alexandrov, P. K. Galenko, A. P. Malygin, and D. M. Kherlakh, "Selection of the steady-state growth mode of the tip of a parabolic dendrite during the forced convection flow and solidification of a binary liquid," Vestn. Udmurt, Univ. 1, 3-16 (2010).

22. D. V. Alexandrov, P. K. Galenko, and D. M. Herlach, "Selection criterion for the growing dendritic tip in a non-isothermal binary system under forced convective flow,” J. Crystal Growth 312, 2122-2127 (2010).

23. B. S. Bokshtein, Diffusion in Metals (Metallurgiya, Moscow, 1978).

24. J. P. Praizey, "Benefits of microgravity for measuring thermotransport coefficients in liquid metallic alloys," Int. J. Heat Mass Trans. 32, 2385-2401 (1989).

25. S. Van Vaerenbergh, J. P. Garandet, J. P. Praizey, and J. C. Legros, "Reference Soret coefficients of natural isotopes and diluted alloys of tin," Phys. Rev. E 58, 1866-1873 (1998).

26. S. Van Vaerenbergh, S. R. Coriell, G. B. McFadden, B. T. Murray, and J. C. Legros, "Modification of morphological stability by Soret diffusion," J. Crystal Growth 147, 207-214 (1995).
27. S. Van Vaerenbergh, S. R. Coriell, and G. B. McFadden, "Morphological stability of a binary alloy: thermodiffusion and temperature-dependent diffusivity," J. Crystal Growth 223, 565-572 (2001).

28. St. Hollinger and M. Lücke, "Influence of the Dufour effect on convection in binary gas mixtures," Phys. Rev. E 52, 642-657 (1995).

29. A. Bruson and M. Gerl, "Diffusion coefficient of Sn, Sb, Ag and Au in liquid Sn," Phys. Rev. B 21, 54475454 (1980).

30. H. E. Huppert, "The fluid mechanics of solidification,” J. Fluid Mech. 212. 209-240 (1990).

31. J. A. Warren and J. S. Langer, "Prediction of dendritic spacings in a directional-solidification experiment," Phys. Rev. E 47, 2702-2712 (1993).

32. Yu. A. Buyevich, D. V. Alexandrov, and V. V. Mansurov, Macrokinetics of Crystallization (Begell House, New York, 2001).

33. V. T. Borisov, V. V. Vinogradov, and I. L. Tyazhel'nikova, "Quasiequilibrium theory of the mushy zone and its application to the solidification of alloys," Izv. Vyssh. Uchebn. Zaved., Chern. Metall., No. 5, 127-134 (1977).

34. R. N. Hills, D. E. Loper, and P. H. Roberts, "A thermodynamically consistent model of a mushy zone," Q. J. Mech. Appl. Math. 36, 505-539 (1983).

35. V. T. Borisov, Theory of the Mushy Zone of a Metallic Ingot (Metallurgiya, Moscow, 1987).

36. D. V. Alexandrov, "On the theory of solidification with a quasiu-equilibrium mushy zone," Dokl. Ross. Akad. Nauk 375 (2), 172-176 (2000).

37. D. V. Alexandrov, "Solidification with a quasiequilibrium mushy zone: exact analytical solution," Int. J. Fluid Mech. Research 27 (2-4), 213-222 (2000).

38. D. V. Alexandrov, "Solidification with a quasiequilibrium mushy region: analytical solution of nonlinear model," J. Crystal Growth 222, 816-821 (2001).

39. D. V. Alexandrov, "Solidification with a quasiequilibrium two-phase zone," Acta Materialia 49, 759-764 (2001).

40. D. L. Aseev and D. V. Alexandrov, "Unidirectional solidification with a mushy layer. The influence of weak convection," Acta Materialia 54, 2401-2406 (2006).

41. D. V. Alexandrov, I. V. Rakhmatullina, and A. P. Malygin, "On the theory of solidification with a two-phase concentration supercooling zone," Rasplavy 4, 88-96 (2010).

42. A. P. Malygin and D. V. Alexandrov, "Analytical description of the quasi-stationary solidification of ternary systems," Rasplavy 6, 11-24 (2011).

43. D. V. Alexandrov, A. P. Malygin, and I. V. Aleksandrova, "Quasi-stationary solidification of ternary systems in the presence of mobile phase transition regions," Vestn. Udmurt. Univ. 2, 12-23 (2011).

44. D. V. Alexandrov and A. P. Malygin, "Self-similar solidification of an alloy from a cooled boundary," Int. J. Heat and Mass Transfer 49, 763-769 (2006).

45. D. V. Alexandrov, "Nonlinear dynamics of solidification of three-component systems," Dokl. Ross. Akad. Nauk 422 (3), 322-326 (2008). 
46. D. V. Alexandrov, A. A. Ivanov, and A. P. Malygin, "On the theory of nonstationary solidification in the presence of a mushy zone," Rasplavy 5, 69-76 (2008).

47. D. V. Alexandrov, A. A. Ivanov, and A. P. Malygin, "Self-similar solidification with a mushy zone from a cooled wall," Vestn. Udmurt. Univ. 1, 14-25 (2008).

48. D. V. Alexandrov, A. A. Ivanov, and A. P. Malygin, "Self-similar solidification of binary alloys," Acta Physica Polonica A 115, 795-799 (2009).

49. D. V. Alexandrov and A. A. Ivanov, "The Stefan problem of solidification of ternary systems in the presence of moving phase transition regions," J. Exp. Theor. Phys. 135, 858-964 (2009).

50. D. V. Alexandrov and A. A. Ivanov, "Analytical solution for a problem of directional solidification in a ternary system," Acta Physica Polonica A 115, 786-790 (2009).

51. D. V. Alexandrov and A. A. Ivanov, "Nonlinear dynamics of directional solidification of ternary solutions with mushy layers," Heat Mass Transfer 45, 1467-1472 (2009).

52. D. V. Alexandrov and A. A. Ivanov, "Solidification of a ternary melt from a cooled boundary or nonlinear dynamics of mushy layers," Int. J. Heat Mass Transfer 52, 4807-4811 (2009).

53. D. V. Alexandrov, A. G. Churbanov, and P. N. Vabishchevich, "Emergence of a mushy region in processes of binary melt solidification," Int. J. Fluid Mech. Research 26 (2), 248-264 (1999).

54. D. V. Alexandrov, "Incipience of a mushy zone in binary melt solidification processes," Int. J. Fluid Mech. Research 27 (2-4), 223-238 (2000).

55. D. V. Alexandrov, "On the theory of the nucleation of a concentration supercooling mushy zone," Dokl. Ross. Akad. Nauk 392, 322-327 (2003).

56. D. V. Alexandrov and A. P. Malygin, "Analytical description of the crystallography of sea water in ice cracks and their effect on the ocean-atmosphere heat exchange," Dokl. Ross. Akad. Nauk 411 (3), 390-394 (2006).

57. D. V. Alexandrov, D. L. Aseev, I. G. Nizovtseva, H.-N. Huang, and D. Lee, "Nonlinear dynamics of directional solidification with a mushy layer. Analytic solutions of the problem," Int. J. Heat Mass Transfer 50, 3616-3623 (2007).

58. D. V. Alexandrov, I. G. Nizovtseva, A. P. Malygin, H.-N. Huang, and D. Lee, "Unidirectional solidification of binary melts from a cooled boundary: analytical solutions of a nonlinear diffusion-limited problem," J. Phys.: Cond. Matt. 20, 114105-01-06 (2008).

59. D. V. Alexandrov, I. G. Nizovtseva, D. Lee, and H.-N. Huang, "Solidification from a cooled boundary with a mushy layer under conditions of nonturbulent and turbulent heat and mass transfer in the ocean," Int. J. Fluid Mech. Research 37 (1), 1-14 (2010).

60. D. V. Alexandrov and I. G. Nizovtseva, "Nonlinear dynamics of the false bottom during the solidification of sea water," Dokl. Ross. Akad. Nauk 419, 262-265 (2008).

61. D. V. Alexandrov and I. G. Nizovtseva, "To the theory of underwater ice evolution, or nonlinear dynamics of "false bottoms," Int. J. Heat Mass Transfer 51, 52045208 (2008).

62. I. V. Alexandrova, D. V. Alexandrov, D. L. Aseev, and S. V. Bulitcheva, "Mushy layer formation during solidification of binary alloys from a cooled wall: the role of boundary conditions," Acta Physica Polonica A 115, 791-794 (2009).

63. D. V. Alexandrov, A. P. Malygin, and I. V. Alexandrova, "Solidification of leads: approximate solutions of nonlinear problem," Annals of Glaciology 44, 118-122 (2006).

64. D. V. Alexandrov, A. V. Netreba, and A. P. Malygin, "On the theory of directional solidification with a phase transition zone under convection and kinetics in a melt," Rasplavy 4, 62-76 (2011).

65. D. V. Alexandrov and A. P. Malygin, "Effect of convection, anisotropy, and heterogeneity of a medium in the structure-phase transitions during solidification," Dokl. Ross. Akad. Nauk 434, 327-331 (2010).

66. D. V. Alexandrov and D. L. Aseev, "One-dimensional solidification of an alloy with a mushy zone: thermodiffusion and temperature-dependent diffusivity," J. Fluid Mechanics 527, 57-66 (2005).

67. D. V. Alexandrov and D. L. Aseev, "Directional solidification with a two-phase zone: thermodiffusion and temperature-dependent diffusivity," Computational Materials Science 37, 1-6 (2006).

68. G. Lamb, Hydrodynamics (OGIZ, Moscow, 1947).

69. N. E. Kochin, I. A. Kibel', and N. V. Roze, Theoretical Hydromechanics (OGIZ, Moscow, 1947), Vol. 2.

70. D. V. Alexandrov and V. V. Mansurov, "Methodical notes on the strong solution of some well-known problems of hydrodynamics," Int. J. Fluid Mech. Research 26, 232-247 (1999).

71. D. L. Aseev and D. V. Aleksandrov, "Nonlinear solidification dynamics of binary melts with a nonequilibrium mushy zone," Dokl. Ross. Akad. Nauk 408, 609613 (2006).

72. D. L. Aseev and D. V. Alexandrov, "Directional solidification of binary melts with a non-equilibrium mushy layer," International J. Heat and Mass Transfer 49, 4903-4909 (2006).

73. D. V. Alexandrov, D. L. Aseev, and A. P. Malygin, "On the theory of the solidification processes with a nonequilibrium mushy zone," Rasplavy 1, 16-30 (2011).

74. D. V. Alexandrov, V. V. Mansurov, and P. K. Galenko, "Morphological stability of the plane interface of the phases in a binary melt during high-rate solidification," Dokl. Ross. Akad. Nauk 351, 37-39 (1996).

75. D. V. Alexandrov and V. V. Mansurov, "Dynamic instability of the quasi-stationary solidification of a binary melt in the presence of a narrow quasi-equilibrium mushy zone," Kristallografiya 41 (2), 376-378 (1996).

76. D. V. Alexandrov and V. V. Mansurov, "Dynamic stability of a solidification process of a binary melt in the presence of a broad quasiequilibrium mushy region," Scripta Materialia 35 (7), 787-790 (1996).

77. D. V. Alexandrov and V. V. Mansurov, "Dynamic instability of the quasi-stationary solidification of a binary 
melt in the presence of a broad quasi-equilibrium mushy zone," Kristallografiya 42 (3), 402-404 (1997).

78. D. V. Alexandrov, "Linear analysis of dynamic instability of solidification with a quasiequilibrium mushy zone," Int. J. Fluid Mech. Research 27 (2-4), 239247 (2000).

79. D. V. Alexandrov, "The effect of concentrational supercooling on the morphological stability of self-similar solidification with a planar front," Doklady Physics 46, 453-458 (2001).

80. D. V. Alexandrov and A. O. Ivanov, "Dynamic stability analysis of the solidification of binary melts in the presence of a mushy region: changeover of instability," J. Crystal Growth 210, 797-810 (2000).

81. D. V. Alexandrov, "A nonlinear instability analysis of crystallization processes with a two-phase zone," J. Metast. Nanocryst. Mater. 20-21, 468-475 (2004).
82. D. V. Alexandrov and A. P. Malygin, "Convective instability of directional crystallization in a forced flow: The role of brine channels in a mushy layer on nonlinear dynamics of binary systems, " Int. J. Heat Mass Transfer 54, 1144-1149 (2011).

83. D. V. Alexandrov and A. P. Malygin, "Convective instability of solidification with a phase transition zone," Zh. Eksp. Teor. Fiz. 139, 688-694 (2011).

84. D. V. Alexandrov and A. P. Malygin, "Coupled convective and morphological instability of the inner core boundary of the Earth," Physics of the Earth and Planetary Interiors 189, 134-141 (2011).

Translated by K. Shakhlevich 